\title{
Novel high temperature vacuum nanoindentation system with active surface referencing and non-contact heating for measurements up to $800^{\circ} \mathrm{C}$
}

Cite as: Rev. Sci. Instrum. 90, 045105 (2019); doi: 10.1063/1.5029873

Submitted: 16 March 2018 - Accepted: 11 March 2019•

Published Online: 2 April 2019

Marcello Conte, ,a, (b) (D) Gaurav Mohanty, 2,3,a) (D) Jakob J. Schwiedrzik, ${ }^{2}$ (D) Jeffrey M. Wheeler, ${ }^{4}$

Bertrand Bellaton, 'Johann Michler, ${ }^{2}$ (D) and Nicholas X. Randall ${ }^{1}$

\begin{abstract}
AFFILIATIONS
${ }^{1}$ Anton Paar TriTec SA, Rue de la Gare 4, Peseux 2034, Switzerland

${ }^{2}$ Laboratory for Mechanics of Materials and Nanostructures, Empa-Swiss Federal Laboratories for Materials Science and Engineering, Feuerwerkerstrasse 39, Thun 3602, Switzerland

${ }^{3}$ Materials Science and Environmental Engineering, Tampere University, 33014 Tampere, Finland

${ }^{4}$ Laboratory for Nanometallurgy, ETH Zurich, Zurich, Switzerland
\end{abstract}

a) M. Conte and G. Mohanty are joint first authors.

${ }^{\text {b) }}$ Author to whom correspondence should be addressed: marcello.conte@anton-paar.com

\begin{abstract}
High temperature nanoindentation is an emerging field with significant advances in instrumentation, calibration, and experimental protocols reported in the past couple of years. Performing stable and accurate measurements at elevated temperatures holds the key for small scale testing of materials at service temperatures. We report a novel high temperature vacuum nanoindentation system, High Temperature Ultra Nanoindentation Tester (UNHT ${ }^{3} \mathrm{HTV}$ ), utilizing active surface referencing and non-contact heating capable of performing measurements up to $800{ }^{\circ} \mathrm{C}$. This nanoindenter is based on the proven Ultra Nano-Hardness Tester (UNHT) design that uses two indentation axes: one for indentation and another for surface referencing. Differential displacement measurement between the two axes enables stable measurements to be performed over long durations. A vacuum level of $10^{-7}$ mbar prevents sample surface oxidation at elevated temperatures. The indenter, reference, and sample are heated independently using integrated infrared heaters. The instrumental design details for developing a reliable and accurate high temperature nanoindenter are described. High temperature calibration procedures to minimize thermal drift at elevated temperatures are reported. Indentation data on copper, fused silica, and a hard coating show that this new generation of instrumented indenter can achieve unparalleled stability over the entire temperature range up to $800{ }^{\circ} \mathrm{C}$ with minimum thermal drift rates of $<2 \mathrm{~nm} / \mathrm{min}$ at elevated temperatures.
\end{abstract}

Published under license by AIP Publishing. https://doi.org/10.1063/1.5029873

\section{INTRODUCTION}

Nanoindentation is an instrumented indentation technique in which controlled loads or displacements are applied on a specimen using a geometrically well-defined tip. The load and the displacement are recorded with high resolution, often with nano-Newton and sub-nanometer precision, respectively. An indentation performed with the tip on the specimen surface is typically comprised of three segments corresponding to loading, holding, and unloading of the indenter tip. From an indent, the mechanical properties of the tested material, e.g., hardness and modulus, are determined by applying relevant contact mechanics solutions ${ }^{1,2}$ and appropriate corrections (such as compliance correction and tip shape determination).

Since emerging in the early $1990 \mathrm{~s},{ }^{1-6}$ nanoindentation has established itself as a routine technique for mechanical property 
measurements, offering a very high degree of flexibility and applicability. It has been used to study the entire spectrum of materials covering metals, ceramics, polymers, composites, biological specimens, etc. By using different tip geometries (e.g., flat punch, conospherical, and cube-corner) and utilizing focused ion beam milled structures such as micropillars and micro-cantilevers, nanoindentation is also being used to study uniaxial compression, bending, and fracture. Considerable advances have been made in the nanomechanics community to include different mechanical properties that can be studied using a nanoindenter. These include yield strength, ${ }^{7}$ fracture toughness, ${ }^{8}$ strain rate sensitivity, ${ }^{9,10}$ stress relaxation, ${ }^{11}$ creep, ${ }^{12}$ low cycle fatigue, impact, high strain rates, interface adhesion strength, viscoelastic properties, etc. It has also been combined with scanning electron microscopy (SEM), ${ }^{13}$ transmission electron microscopy (TEM), ${ }^{14-16}$ high resolution electron backscatter diffraction (EBSD), ${ }^{17}$ Raman, ${ }^{18}$ synchrotron x-rays, ${ }^{19}$ and light microscopes to visualize the deformation in situ and correlate the mechanical property data with the evolving structural/ crystallographic/microstructural information. Recently, its extension to non-ambient conditions such as high ${ }^{20-22}$ and cryogenic temperatures, ${ }^{23,24}$ humid environments, ${ }^{25}$ and liquid cell testing ${ }^{26}$ of biological samples has enabled investigation of material deformation behavior in actual service conditions.

High temperature nanoindentation enables studying not only material properties at elevated temperatures but also the fundamental rate controlling deformation mechanisms in materials which could greatly aid alloy design efforts. The extension of nanoindentation to elevated temperatures is not straight forward, particularly if a conventional nanoindenter is heated without necessary modifications. The thermal expansion of the tip and load frame components due to heating can potentially be in the range of microns, completely overshadowing the nanoscale displacements that are being studied. These thermal expansion data get convoluted with the mechanical deformation behavior of the sample being probed. Additionally, oxidation of the sample surface, tip-sample reactions, tip wear, blunting, and contamination are other important issues. Although there have been efforts to extend nanoindentation to elevated temperatures over the past two decades, it is only recently that substantial breakthroughs have been achieved in terms of performing reliable and accurate measurements. This has come about through important incremental steps-tip and sample heating, ${ }^{20}$ introduction of inert gas environment/partial pressure ${ }^{27}$ or high vacuum $^{28}$ to prevent oxidation, determining isothermal contact conditions, ${ }^{29}$ tip-sample surface temperature matching to minimize thermal drift, ${ }^{30}$ and finally, minimizing tip material wear ${ }^{31}$ and tipsample reactions. ${ }^{32}$ Concurrently, various associated instrumentation technologies, calibration methods, and experimental protocols have been reported, an overview of which can be found here. ${ }^{22,33-35}$ There has been an upward trend in the highest test temperatures reported in nanoindentation using various instruments ${ }^{36-40}$ off late.

The majority of existing instruments/prototypes report the use of resistive heating. While this is not a limitation in itself, the stiffness exerted by the thicker resistive heating wires (in comparison with the thin thermocouple wires) decreases the load/displacement resolution of the technique compared to room temperature testing. Since free movement of the indenter tip is at the heart of nanoindentation, any obstruction from the wires can result in decreased data resolution, if not erroneous data and artefacts of measurement. The stiffness of these wires also changes with temperature which has to be considered. Sometimes, the user has to spend substantial time and effort in ensuring the free movement of the indentation axis/indenter head/tip. In addition, from an operational point of view, any breakdown in the heating elements using contact resistive heating requires extensive repair and careful maintenance. Therefore, most high temperature nanoindentation systems are fragile and require highly skilled users and large investments in time and effort for testing compared to standard room temperature operation. This is one of the important factors that currently limits the widespread acceptance and use of high temperature nanoindentation.

Another limitation with current instrumentation is the lack of long term stability for performing measurements such as nanoindentation creep. Beyond a certain time of measurement, typically $5 \mathrm{~min}$, the thermal drift of the system catches up with the nanomechanical deformation data. This has been addressed, up to some extent, by utilizing sinus mode indentation and back calculating and correcting for the thermal drift assuming constant modulus of the sample. ${ }^{41}$ However, this requires precise information about the material properties a priori either from the literature or from fast high temperature measurements. It is therefore an objective to have a system that enables low drift and stable high temperature measurements for such studies directly.

This work aims to overcome these challenges by reporting the development of a novel high temperature vacuum nanoindentation system that uses non-contact heating and active surface referencing to minimize thermal drift. The design considerations and instrumental details for this new high temperature nanoindentation system will be presented in this work. Experimental protocols for thermal drift minimization using this instrument will be reported and the extracted mechanical properties of standard materials will be compared with literature values to validate the developed high temperature nanoindentation system.

\section{INSTRUMENTATION}

A novel high temperature nanoindentation system, High Temperature Ultra Nanoindentation Tester (UNHT $\left.{ }^{3} \mathrm{HTV}\right)$, capable of performing measurements up to $800{ }^{\circ} \mathrm{C}$ in vacuum has been developed. This system is based on the proven Ultra Nanoindentation Tester $\left(\mathrm{UNHT}^{3}\right.$ ) indentation head. ${ }^{42}$ The maximum load and displacement ranges are $100 \mathrm{mN}$ and $100 \mu \mathrm{m}$, respectively, with corresponding theoretical digital resolutions of $6 \mathrm{nN}$ and $0.006 \mathrm{~nm}$. The noise floor provides a more practical idea of the instrument performance in a laboratory environment. The root mean squared (RMS) load and displacement noise floor in ambient conditions (room temperature operation) are $0.5 \mu \mathrm{N}$ and $0.15 \mathrm{~nm}$, respectively. The RMS noise floor for load and displacement increases to $2 \mu \mathrm{N}$ and $0.4 \mathrm{~nm}$, respectively, when testing at elevated temperatures in vacuum. However, it is not a customized version of the standard ultra nano-hardness tester (UNHT) nanoindenter; rather it incorporates substantial design and development to enable high temperature vacuum nanoindentation. The system consists of independent non-contact tip and sample heating using infrared for elevated temperature measurements. The important features of the developed high temperature nanoindentation system are described in this section. 


\section{A. Active surface referencing}

It will be useful to discuss the principle of $\mathrm{UNHT}^{3}$ operation as it is critical for subsequent discussion on high temperature nanoindentation protocol development. The principle of $\mathrm{UNHT}^{3}$, unlike most nanoindentation systems, is based on the idea of using two independent and symmetric vertical axes: one for indentation and the other for active surface referencing. Both the axes have their own independent actuators and their own displacement and load sensors (Fig. 1). Force is applied on each axis by piezoelectric actuation $\left(A_{1}\right.$ and $\mathrm{A}_{2}$ ) through springs of calibrated stiffness value $K$. Note that the indenter and the reference axes are identical in terms of mechanical design including having the same spring constant $K$. The piezoelectric actuators $\left(A_{1}\right.$ and $\left.A_{2}\right)$ are used for actuation, and the force is measured through the capacitors $\mathrm{C}_{1}$ and $\mathrm{C}_{3}$ for the indenter and the reference, respectively. The force is controlled on each axis using independent feedback control loops between the respective capacitors and the piezoelectric actuators (e.g., $C_{1}$ and $A_{1}$, and $C_{3}$ and $A_{2}$ ). Force is detected only when the indenter (and the reference) is in contact with the sample. The differential displacement between the reference, which sits on the sample surface, and the indenter, which performs the indentation, is measured through capacitor $\mathrm{C}_{2}$ which bridges the two vertical axes. The reference has a spherical geometry with a large diameter of $1.5 \mathrm{~mm}$ that results in negligible penetration by the reference into ceramic or metallic samples when applying small loads (typically $1-5 \mathrm{mN}$ ). Any mechanical vibrations, if at all, are experienced by both the indenter and the reference. These vibrations are attenuated due to differential displacement measurement and symmetric mechanical design of both axes. This is at the heart of active surface referencing technology that results in stable measurements over long test durations. The symmetric architecture of the $\mathrm{UNHT}^{3}$ configuration comprising an indenter and a reference axis, active surface referencing during indentation, and use of extremely low coefficient of thermal expansion materials such as Zerodur and



FIG. 1. Schematic of the UNHT ${ }^{3}$ HTV configuration comprising two independent vertical axes for active surface referencing: one for indentation (left) and the other for surface referencing (right). Force is applied by piezoelectric actuation (A1 and A2) through springs (K). The force is measured from the capacitors $\mathrm{C} 1$ and $\mathrm{C} 3$ for the indenter and the reference, respectively, using independent feedback control loops. The differential displacement between the reference and the indenter is measured from capacitor C2 which bridges the two vertical axes. Infra-red (IR) heaters and location of thermocouples are also depicted.
Macor results in low frame compliance and low thermal drift at room temperature. A detailed description of the UNHT concept and its room temperature operation characteristics have been reported by Nohava et al. ${ }^{43}$

The distance between the reference and the indenter axis is $9.25 \mathrm{~mm}$ (compared to $1.5 \mathrm{~mm}$ for the standard $\mathrm{UNHT}^{3}$ system). This is due to the requirement for extra space for tip heating. For samples smaller than $1 \mathrm{~cm}$ in diameter/length, two identical samples of similar thicknesses can be used for measurements-one for indentation and another for landing the reference. Owing to the large indentation depth range of $100 \mu \mathrm{m}$, slight differences in sample thicknesses can be easily accommodated. Unlike other nanoindentation systems, the $\mathrm{UNHT}^{3}$ head is neither a purely load nor a purely displacement controlled instrument. In the case of loadcontrolled tests, the capacitor $\mathrm{C}_{1}$, that measures the load, is on a feedback loop with the actuation applied through the piezoelectric actuator $A_{1}$. For depth controlled tests, the capacitor $C_{3}$ is on a feedback loop with the piezoelectric actuator $A_{1}$. It should be pointed out that the changes in the capacitance of $\mathrm{C}_{1}, \mathrm{C}_{3}$, and $\mathrm{C}_{2}$ are calibrated to measure the indenter load, reference load, and depth of penetration, respectively. Hence, both load and displacement controlled tests can be performed easily using this system. It is also easy to switch between the modes in a single experimental profile, e.g., change from a load controlled test to a displacement controlled test immediately or vice-versa. The user has the option of adding all kinds of load or displacement profiles-linear, proportional, and/or sinus oscillations while setting up an experiment. In addition, grid indents of huge matrices or site specific indents on locations chosen from the optical microscope can also be set up easily. The flexibility provided in the experimental profile and indentation grid setup enables performing all kinds of measurements reliably using the $\mathrm{UNHT}^{3}$ head-indentation, microcompression, fracture, creep, etc.

\section{B. Vacuum system}

Material surface oxidation is particularly undesirable as nanoindentation is a surface mechanical property measurement technique. Previous approaches to alleviate this problem have included application of thin noble metal coatings on the surface and use of inert/reducing environments, partial vacuum, and high vacuum (like in SEM, normally $10^{-6}$ mbar). Volinsky et al. ${ }^{44}$ used a $5 \mathrm{~nm}$ thin Pt layer on a sputtered $\mathrm{Cu}$ film to prevent oxidation up to $130{ }^{\circ} \mathrm{C}$. However, this strategy cannot be used on all materials and for high temperatures due to diffusion and surface property modification. Inert environments and partial vacuum might not be suitable for highly reactive metals. ${ }^{22}$ Reducing environments can potentially affect the instrument and cause hydrogen embrittlement of indenter components. This highlights the importance of "good" vacuum levels for high temperature testing.

Figure 2 shows a schematic of the instrument with the vacuum chamber and pumping systems incorporated. The high vacuum system comprises an enclosure, a pumping station, and a series of valves and gauges to control the pressure in the chamber. The UNHT ${ }^{3}$ HTV system is fixed on a frame specially designed to damp vibrations. It is integrated in a vacuum chamber, with a capacity of about 100 liters, which is suspended on active air pads for vibration isolation. The pumping station is composed of a primary roughing pump and a secondary turbo-molecular pump. The latter is separated from 


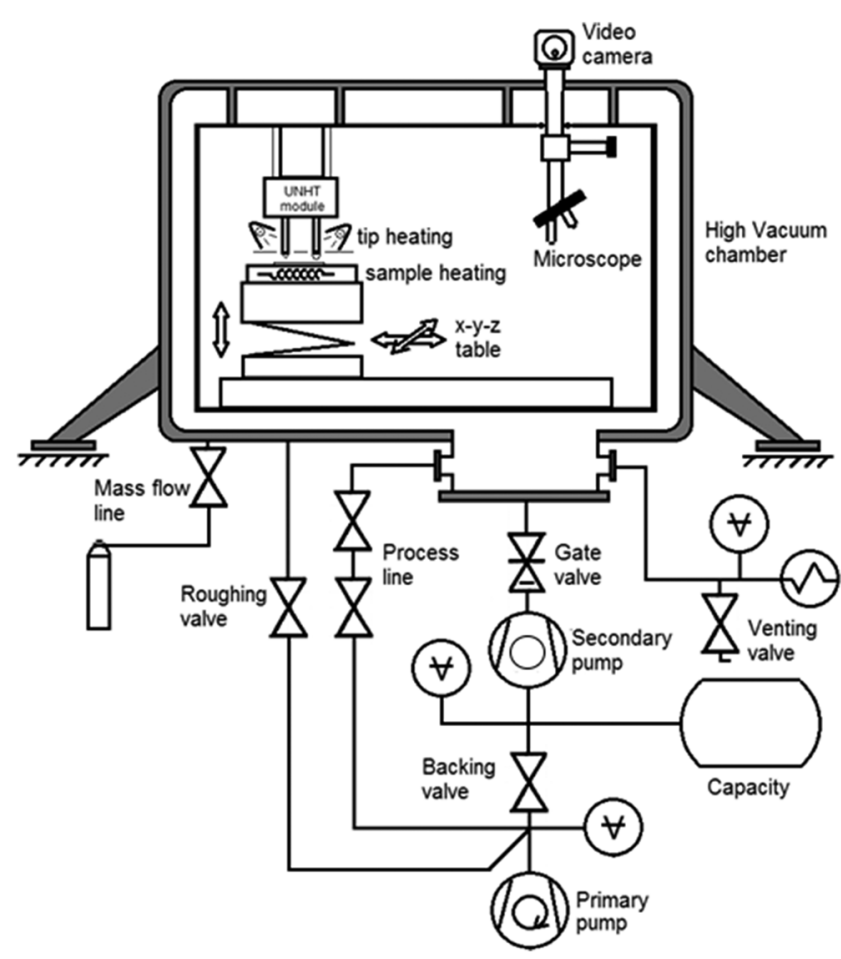

FIG. 2. Schematic of the vacuum high temperature nanoindentation tester $\left(\mathrm{UNHT}^{3}\right.$ HTV).

the chamber by means of a gate valve and is used to pump the chamber down to the desired vacuum level. The chamber can be evacuated to vacuum levels of $10^{-7}$ mbar. Various gauges and valves are used to control the system using a programmable logic controller (PLC). There is the possibility of attaching gas supply lines $\left(\mathrm{N}_{2}, \mathrm{Ar}\right.$, etc.) for backfilling the chamber. This allows repeated sequential evacuation and purging of the chamber with inert gases, such as Ar, to reduce surface oxidation of highly reactive metals for high temperature studies. The system can also be used in inert or nitrogen gas atmosphere and in partial vacuum using only the roughing pump $\left(10^{-3}\right.$ mbar).

Vacuum operation invariably introduces additional mechanical noise into the measurements due to operation of the pumps and valves. Therefore, specific design changes have been made to minimize perturbations from affecting the nanoindentation measurements. First and foremost, the pumps need to be mechanically isolated from the chamber. A 5-axis, magnetically levitated, turbomolecular pump is used to minimize mechanical vibrations emanating from the pumping system. The entire vacuum chamber is mounted on a 4-point anti-vibration table that uses active compressed air to float the chamber. In addition, the vacuum chamber has been purposefully designed to be heavy to attenuate vibrations. Apart from this, the measurement head itself is mounted on a specially designed frame inside the vacuum chamber which has been optimized by using a combination of cast iron, aluminum, and stainless steel, permitting optimum mechanical damping. To reduce the noise emanating from the primary roughing pump, a vacuum buffer has been introduced between the secondary pump and the backing valve. This cuts down the need for running the roughing pump all the time and allows the chamber vacuum to be buffered at $10^{-6}$ mbar, typically for over $30 \mathrm{~h}$ at a time. Finally, active surface referencing further reduces mechanical noise. Any potential perturbation/mechanical vibration transmitted to the sample is experienced by both the reference and the indenter. This gets attenuated due to the differential displacement measured between the reference and the indenter allowing stable long term measurements without artefacts. The vacuum system has been designed using a modular approach by dividing it into mechanical, vacuum, heating, and cooling sub-systems. ${ }^{45}$ The efficiency of the various sub-systems was independently assessed, and any problems arising from their interactions were resolved.

\section{Indenter tip heating}

The tips, both the indenter and reference, are heated independently using non-contact infrared heating sources. Halogen tungsten lamps of $150 \mathrm{~W}$ each are used for heating and are located at the first focal point of an ellipsoidal mirror, coated with a reflective gold coating. The reflective coating limits the absorption of the infrared radiation by the mirror and maximizes the heating efficiency. The indenter and reference tips are placed at the second focal point ${ }^{46}$ of the ellipsoidal mirror. The reflector surface is substantially ellipsoidal which results in even heating of the indenter tip without any local hotpots. This is a substantial improvement over the use of conventional contact resistive heating in other nanoindentation systems. Typically, resistive wires are thicker in comparison with thermocouple wires and inhibit the movement of the indenter. Noncontact heating ensures that the indenter and the reference are free to move without these restrictions. The high temperature tip assembly is longer compared to standard room temperature tip design. It should be noted that by tip assembly, we refer to the indenter shaft and the diamond tip together. The tip assembly has been specially designed for high temperature operation by optimizing efficient heat absorption from the infrared heaters and heat insulation to protect the sensors in the indentation head. Consequently, the tip assembly comprises a lower infrared absorber part made of molybdenum and an upper heat insulator part made of Macor. ${ }^{47}$ Diamond (and other materials such as tungsten carbide and cubic boron nitride) tips are clamped to the bottom part of the tip assembly. For temperature monitoring of both the indenter and the reference, thin N-type thermocouples (about $75 \mu \mathrm{m}$ diameter) pass through the tip assembly and are located close to the tips. The thermocouples have a temperature resolution less than $0.1{ }^{\circ} \mathrm{C}$ and have fast responses of less than $0.01 \mathrm{~s}$ to temperature changes. Both indenter and reference tips are heated separately and are regulated independently by means of a proportional integral (PI) control editable by the user. During measurements, an infrared environment is created on top of the sample that drastically reduces heat losses and aids in thermal stabilization, as shown in Fig. 3. The use of a high vacuum environment helps contain the infrared "bath" as convective losses are minimized.

\section{Sample mounting and heating}

Sample mounting for high temperature measurements is not straightforward. Room temperature measurements typically use glue 


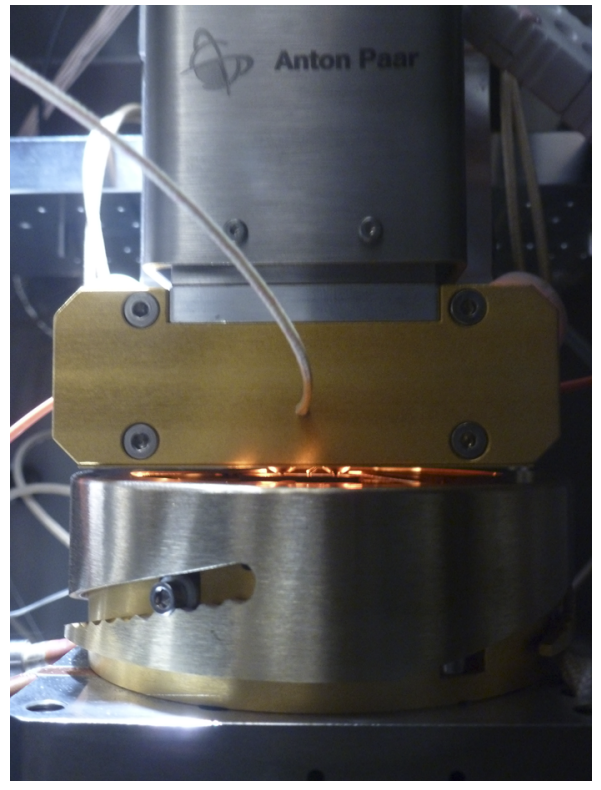

FIG. 3. Image showing the UNHT ${ }^{3}$ HTV system through the vacuum window.

(cyanoacrylate, epoxy glues, etc.) to fix the sample onto the sample holder. These are not suitable for high temperature use. A variety of cements have been used in the past for high temperature tests. These cements often require a drying and curing step before use at elevated temperatures. ${ }^{22}$ Another limitation of using cements is that they are prone to thermal shocks and can crack at elevated temperatures. This often results in the sample coming loose in the middle of the tests and/or can contribute to additional sample compliance which may produce error in the measured mechanical properties. Sample gluing is therefore not a dependable solution and increases the time of high temperature nanoindentation tests. One solution suggested in the literature is mechanical clamping. ${ }^{22,27}$ Simple clamping using screws and jaws can lead to relative movements of the parts due to thermal expansion and unnecessary heat dissipation from the sample. This limits the heating efficiency and the maximum attainable temperature for a certain power output.

To overcome these issues, a high temperature sample holder was specially designed. The sample heating stage ${ }^{48}$ incorporates several innovative design features compared to the other similar existing systems. To avoid turbulent water flow through the cooling tubes, a laminar flow stabilizer is used (refer to Sec. III A). The sample is placed on a rigid thermally conductive disc and clamped from the top by means of a retaining ring. The top retaining ring has radial extensions of different lengths to accommodate samples of different widths (or diameter). Radial extensions are preferable over using radial rings so as to minimize the contact area and, consequently, heat losses from sample surface by conduction. The retaining rings are engaged with notches that allow placing them in three symmetric positions, $120^{\circ}$ from each other, to accommodate various sample geometries and thicknesses. The sample is mounted on a holder disc that is free to rotate before being locked and is placed on three insulating cylindrical supports. Two of these cylindrical supports are
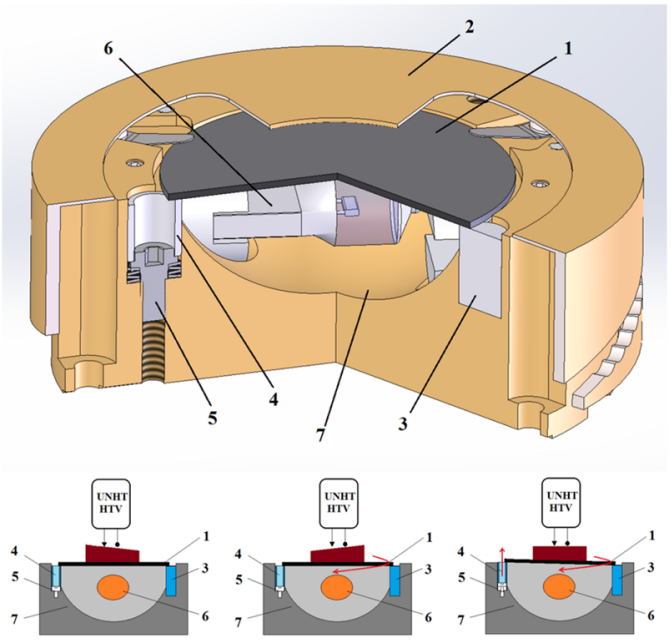

FIG. 4. Sample heating stage section. The sample is placed on the graphite disc (1) and clamped by means of the retaining ring (2). The former is placed on two fixed supports (3) and one adjustable support (4). Acting on the screw (5) and turning the disc (1), it is possible to have the sample surface orthogonal to the indenter. The disc (1) is heated up by means of a radiation element (6) placed in a spherical reflector (7).

fixed, while one is adjustable in terms of height (refer to Fig. 4). The sample holder can accommodate fairly large specimens, up to $50 \mathrm{~mm}$ in diameter. The adjustable support can be used to change the tilt of the specimen. This is an important feature in the case of a top active referencing where a slanted specimen can result in the indenter tip touching the specimen surface before the reference tip. By adjusting the tilt of the sample using the adjustable support, specimens having non-parallel faces can be used for indentation by allowing the specimen surface to remain orthogonal to the indenter tip axis. The principle of the sample alignment using this sample stage is shown in Fig. 4.

The sample is heated by two halogen lamps ( $150 \mathrm{~W}$ each) that are placed below the conductive disc made of graphite. Regardless of the sample dimensions and thermal properties, the conductive disc acts as thermal mass that stabilizes the temperature evenly. A concave mirror (spherical) at the bottom directs the infrared rays uniformly onto the conductive disc for uniform heating similar to the tip heating setup described earlier. The infrared heaters are optimized for fast sample heating (typically $50{ }^{\circ} \mathrm{C} / \mathrm{min}$ ). A thermocouple touching the conductive holder disc from the bottom is used to read the sample temperature. Since the sample surface temperature will be different from the temperature read by the bottom thermocouple, an additional optional thermocouple can be placed between the indenter and the reference tip. During indentation, this thermocouple remains in contact with the sample surface and provides a "true reading" of the sample surface temperature.

The sample holder design is modular. The conductive discs and the samples can be changed easily allowing a faster turnaround time between tests on different samples. The infrared sources (from the sample, indenter, and reference heaters) are less prone to breakdowns, a common issue in high temperature nanoindentation testing with resistive heaters. 


\section{E. Thermal management}

The success of high temperature nanoindentation depends on thermal management of the system. The key point is to limit the heating to as small a volume as possible and prevent the heat from affecting the actuators, sensors, and electronics. This is accomplished by using an optimized water cooling design for the indentation head and the sample stage, increasing the heating efficiency by employing a gold coated heat shield on the indentation head facing the tips to reflect maximum infrared radiation toward the sample/tips and, finally, by using a tip design comprising thermal insulation at the upper part in order to minimize heat transfer to the indentation head. All of these features result in efficient thermal management of the system. The indentation head temperature, very close to the piezoelectric actuators, reaches only $28{ }^{\circ} \mathrm{C}$ while performing measurements at $800{ }^{\circ} \mathrm{C}$. This is only $6{ }^{\circ} \mathrm{C}$ higher than room temperature vacuum operation with water cooling. The overall cooling efficiency is greater than $95 \%$ due to these improvements. The various technologies of the tip heating, sample heating system, and sample holder are covered by three filed patents. ${ }^{46-48}$

\section{SYSTEM CHARACTERIZATION}

\section{A. Noise}

Advances in instrumentation in recent years have resulted in lower noise floor and more effective vibration isolation of nanoindentation systems compared to the previous two decades. However, the use of a vacuum environment for high temperature operation will introduce additional mechanical noise in spite of the design improvement mentioned in Secs. II A-II E. The main sources of noise, in this case, will be the primary and the secondary pumps. It is therefore necessary to characterize the noise in nanomechanical measurements experimentally. The high sensitivity of the $\mathrm{UNHT}^{3}$ HTV itself can be used to compare noise levels for different vacuum operating modes.

Noise is typically correlated with standard deviation in experimental data. For a certain mean signal (force or displacement), higher standard deviation corresponds to higher noise levels. Therefore, to experimentally compare the relative noise levels in measurements performed in various operating conditions, indentation tests were performed on fused silica $\left(\mathrm{SiO}_{2}\right)$ using a Berkovich tip at room temperature. The indentation profile comprised of sequentially loading to $10 \mathrm{mN}$ in $10 \mathrm{~s}$, holding the peak load for $15 \mathrm{~s}$, unloading to $0.5 \mathrm{mN}$ in $10 \mathrm{~s}$, holding the drift hold segment load of $0.5 \mathrm{mN}$ for $300 \mathrm{~s}$, and finally unloading completely (see Fig. 5). The data acquisition rate was $20 \mathrm{~Hz}$. The standard deviation of the displacement data recorded during the thermal drift hold segment at $0.5 \mathrm{mN}$ can be used to compare the relative noise levels for four different operating conditions-ambient, primary vacuum $\left(\sim 10^{-3} \mathrm{mbar}\right.$, roughing pump), secondary vacuum $\left(\sim 10^{-6} \mathrm{mbar}\right.$, turbo pump), and measure mode $\left(\sim 10^{-6}\right.$ mbar, turbo pump without the roughing pump). The measure mode refers to the silent operating mode whereby a vacuum buffer is used to run the turbo pump instead of the roughing pump. It should be noted that these tests were conducted after waiting for approximately $30 \mathrm{~min}$ under each pressure condition to allow time for thermal and mechanical stabilization. A variation in depth of $<1 \mathrm{~nm} / \mathrm{min}$ was recorded during the drift hold segment for all pressure modes suggesting excellent thermal drift stability. The standard deviation in depth data was $1.2 \mathrm{~nm}$ in ambient condition, $0.7 \mathrm{~nm}$ in primary vacuum, $5.3 \mathrm{~nm}$ in secondary vacuum, and $3.4 \mathrm{~nm}$ in measure mode. Due to the absence of roughing pump, measure mode indentation has lower noise compared to the secondary and rough pumps together. These experiments validate the efficiency of the instrumental design modifications outlined in Sec. II B to allow stable measurements in high vacuum levels $\left(10^{-6}\right.$ mbar $)$.

Water circulation is an efficient way to eliminate the excess heat from the transducers and sensors. However, water circulation in the indentation head itself can introduce additional noise due to turbulent flow. A laminar flow stabilizer was added to the water cooling tubes to minimize noise in the experimental data. To experimentally verify the effect of addition of laminar flow stabilization on relative noise levels, similar indentation profiles and data analysis, as mentioned in previous paragraph, were performed on sapphire for different water flow rates with and without the laminar flow stabilizer. Figure 6 plots the standard deviation in hold segment displacement data for the two conditions. Displacement standard deviation is drastically reduced with the introduction of laminar flow and does not change much with increase in water flow rate. The fact that the displacement standard deviations are comparable to those without water cooling highlights the efficiency of the laminar flow stabilizer. These two solutions-backing reservoir for the secondary pump and laminar flow stabilizer for the water cooling-result in high

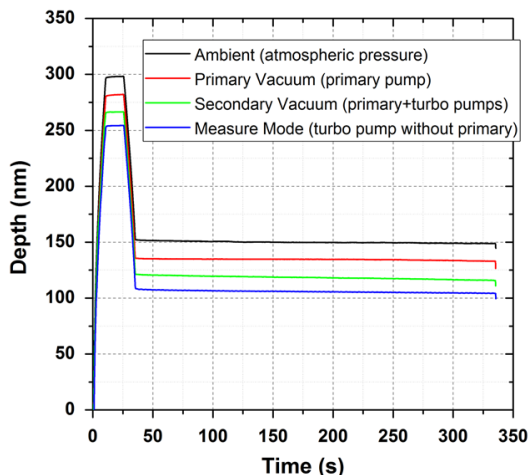

(a)

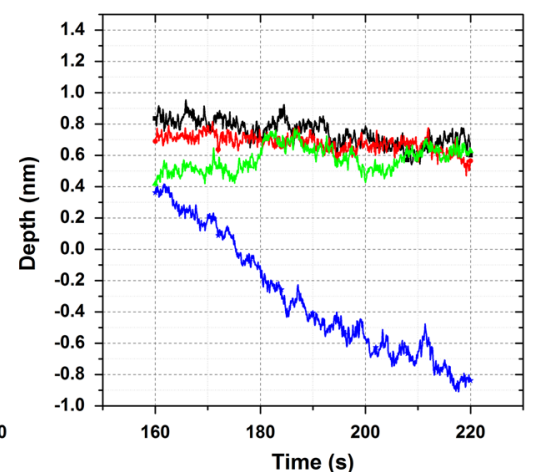

(b)
FIG. 5. Stability tests on fused silica at room temperature in different vacuum regimes. Acquisition frequency $20 \mathrm{~Hz}$. (a) Depth-time curves for the stability tests. The curves are shifted in depth for better differentiation. (b) Depth-time curves for the hold period from (a) are shown. The depth values are normalized to start from zero. On the right, an extract of $60 \mathrm{~s}$ holding segment is shown. 


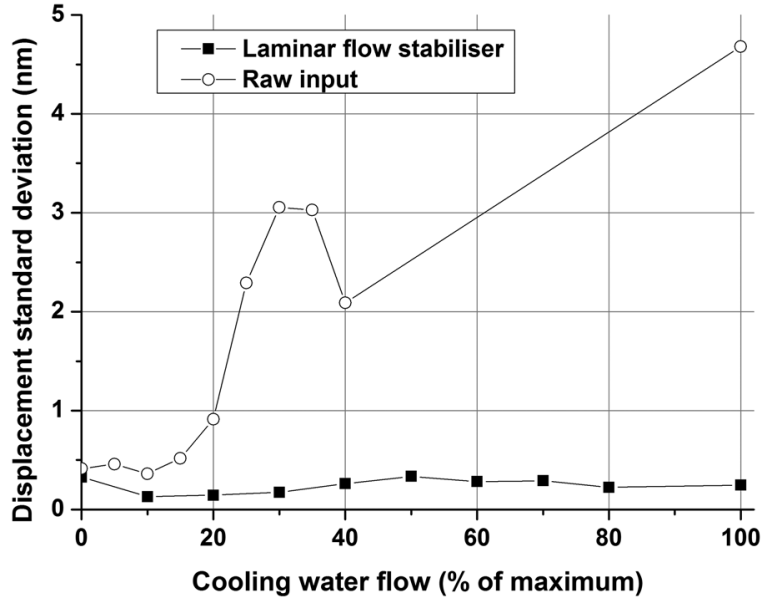

FIG. 6. Effect of water flow on nanoindentation displacement measurements.

temperature performance comparable to standard room temperature operations in terms of relative noise levels.

\section{B. Thermal stabilization time}

One critical aspect of high temperature operation is the thermal stabilization time of the nanoindentation system. It determines how long the operator has to wait after heating (or changing the temperature) before commencing the intended experiments. This is also tied intrinsically to the question of "pre-contact drift." Also referred to as "frame drift," ${ }^{21,30}$ it refers to the thermal expansion of the indenter tip, components in the loading axis, and the indenter frame before contact. It can be considered to be solely occurring in and confined to the load frame and the indenter tip. In other words, it is the preexisting thermal expansion/contraction occurring due to insufficient thermal stabilization.

Use of zero thermal expansion materials for constructing the loading axis and providing sufficient stabilization time, taking into account the thermal mass and thermal responsiveness of the system, are necessary for minimizing pre-contact drift and the thermal stabilization time. Therefore, it is necessary to determine the thermal stabilization time for pre-contact drift to subside to sufficiently low levels. To test this out, the worst case scenario for heating the indentation head was tried out involving the longest heating paths and highest head temperatures. The sample was positioned within $1 \mathrm{~mm}$ of the indenter tip surface and was heated to $200{ }^{\circ} \mathrm{C}$ in air rapidly at $20{ }^{\circ} \mathrm{C} / \mathrm{min}$ without water cooling. On reaching the target temperature, the sample temperature was held constant. This resulted in an indentation head temperature of $42{ }^{\circ} \mathrm{C}$, much higher than the $28{ }^{\circ} \mathrm{C}$ experienced by the head when performing experiments at $800^{\circ} \mathrm{C}$ in vacuum and with water cooling.

Figure 7 shows the evolution in load and displacement signals for this extreme scenario. The indenter and the reference load signals stabilize within 20 min of reaching the target temperature. Differential displacement drift rate, computed from the slope of depth signal with time and by incorporating the appropriate calibration factor for conversion, drops down to less than $4 \mathrm{~nm} / \mathrm{min}$ within an hour and to values less than $2 \mathrm{~nm} / \mathrm{min}$ within $2 \mathrm{~h}$ of stabilization.



(b)

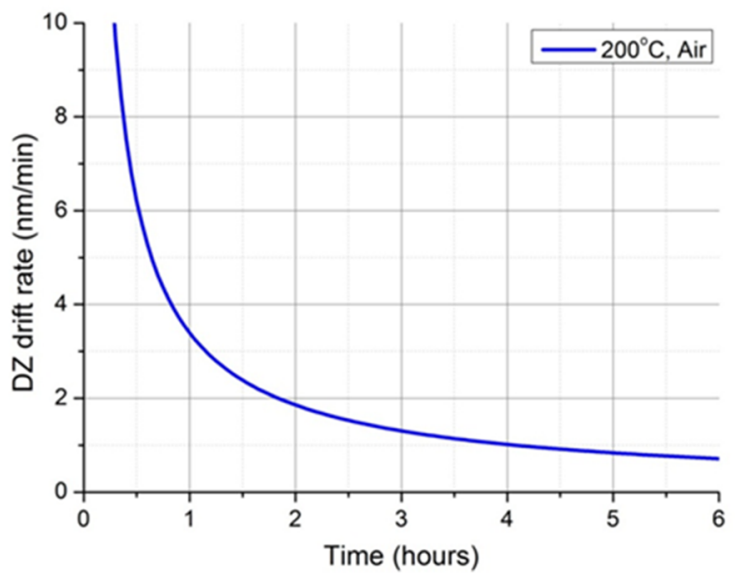

FIG. 7. (a) Pre-contact load and displacement variation for extreme heating scenario for the instrument. Fnl and FnR correspond to force sensor readouts of the indenter and reference, respectively. DZ corresponds to differential displacement readout. The load and displacement signals stabilize within an hour of heating. (b) Displacement drift rate calculated from (a).

Since actual experimental conditions are milder compared to this extreme scenario, it was safely concluded that the maximum thermal stabilization time for the $\mathrm{UNHT}^{3} \mathrm{HTV}$ is less than an hour for the most extreme case of experimental heating, i.e., from room temperature to $800{ }^{\circ} \mathrm{C}$. Quick thermal stabilization time enables the testing of multiple temperatures in a single day and makes high temperature nanoindentation a truly high throughput technique for nanomechanical characterization.

\section{THERMAL DRIFT MINIMIZATION PROTOCOL}

Minimizing thermal drift is necessary for reliable and accurate high temperature measurements. High temperature calibration and indentation protocols play an important role in minimizing drift. Previous work on thermal drift minimization has reported on determining isothermal contact based on thermal drift measurements $^{29}$ and temperature shifts measured by the indenter tip 
thermocouple. ${ }^{21,30}$ This work builds on these previous approaches and adapts them to the current instrumentation.

\section{A. Thermal drift}

Before proceeding further, it is necessary to understand the various contributions of thermal drift. Thermal drift can be divided into three major components depending on their origin: (a) pre-contact drift or frame drift, (b) contact drift, and (c) electronic drift.

Pre-contact drift was addressed in Sec. III B. Contact drift arises due to thermal mismatch between the tip and the sample. This results in temperature changes in both on contact-one expands on gaining heat and the other contracts. Contact drift can therefore be considered to arise solely during indentation/compression. The direction of contact drift depends on whether the indenter is hotter or colder in comparison with the sample surface. A hotter indenter tip will contract during indentation, and the depth sensors will read an apparent increase in indentation depth. Hence, it will result in a positive drift, i.e., an increase in depth. For the opposite case, namely, a colder tip, the contact drift will be negative resulting in decreasing depth. The evolution of contact drift with time will depend on the indenter tip shape and the loading profile used. For example, it will most likely be non-linear for Berkovich and pyramidal tip geometries due to the change in surface contact areas with increasing penetration depths, efficiency of heat transfer across the contacting interface, thermal conductivities and capacities of the two contacting bodies and, finally, the type of strain rate used-constant loading rate vs constant strain rate loading rate. For short duration indents, close to the isothermal conditions and for ease of data analysis, we consider the thermal drift to be linear. Please note that this assumption will not hold for long duration tests. The only way to minimize contact drift is to match the temperatures of the indenter tip and the sample surface as closely as experimentally possible before contact, which will be discussed in Sec. IV B.

The third kind of drift is electronic drift. It does not arise due to physical expansion/contraction; rather its source is the drift in signals due to the sensors affected by heat. This has been addressed by moving the sensors and electronics further away from the heat source, robust cooling of the indentation head/sensors/electronics, and use of electronic components that are stable in the operating temperature range experienced by them. Efficient heat management and electronic stability are the only known methods to minimize electronic drift which have already been addressed in the case of the $\mathrm{UNHT}^{3}$ HTV system.

\section{B. Contact drift minimization}

The temperatures read by the indenter, reference, and sample thermocouples do not represent the actual temperatures of the contacting bodies. There is a temperature gradient from where the temperature is measured to that of the actual contacting surfaces (indenter tip apex and sample surface). Since the indenter (and consequently, the reference as well) is a smaller thermal mass compared to the sample and the thermocouple is placed closer to the indenter tip, the indenter thermocouple can be used to measure small changes in indenter temperature on contact. ${ }^{21,30}$ By cycling the indenter temperature, performing indents and finding the indenter temperature corresponding to negligible temperature change on contact, the isothermal condition for the two contacting bodies can be determined.

Figure 8(a) shows the temperature change measured by the indenter thermocouple for three different temperatures during indentation on copper. The experimental protocol comprised of load controlled indents with the following segments: (a) linear loading of $5 \mathrm{mN} / \mathrm{s}$ up to $100 \mathrm{mN}$, (b) peak hold at $100 \mathrm{mN}$ for $30 \mathrm{~s}$, (c) linear unloading of $5 \mathrm{mN} / \mathrm{s}$ down to $2 \mathrm{mN}$, (d) hold load at $2 \mathrm{mN}$ for $2 \mathrm{~min}$, and finally, (e) linear unloading at $5 \mathrm{mN} / \mathrm{s}$. The sample and the indenter heating powers were kept constant during these tests. It is necessary to use constant power mode (or fixed heating power) to detect these temperature changes as opposed to the constant temperature mode that introduces fluctuations due to the proportionalintegral-derivative (PID) control loop itself. These experiments were performed by fixing the initial distance between the indenter and the sample surface to $500 \mu \mathrm{m}$ to establish a stable infrared thermal bath. The indentation is comprised of an "indenter approach segment" in which the indenter approaches the sample surface followed by the actual indentation. Specific to the UNHT ${ }^{3}$ HTV design that utilizes

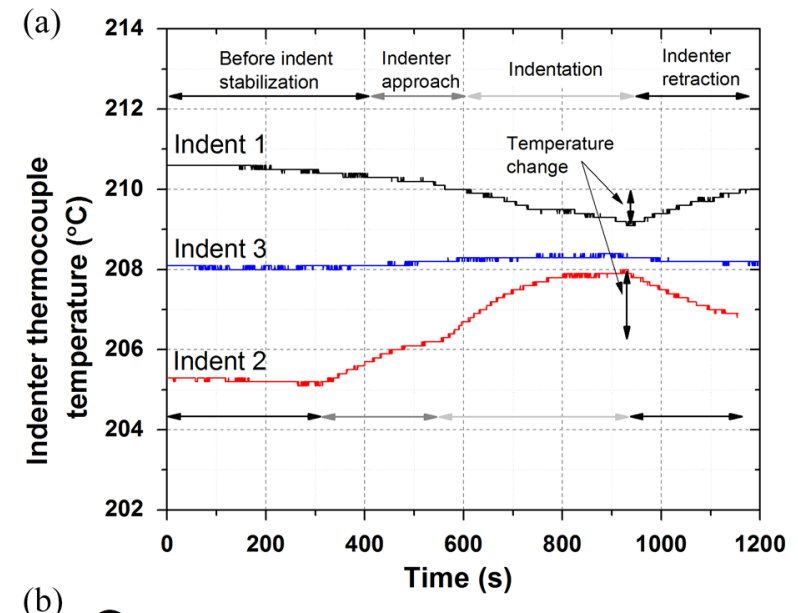

(b)

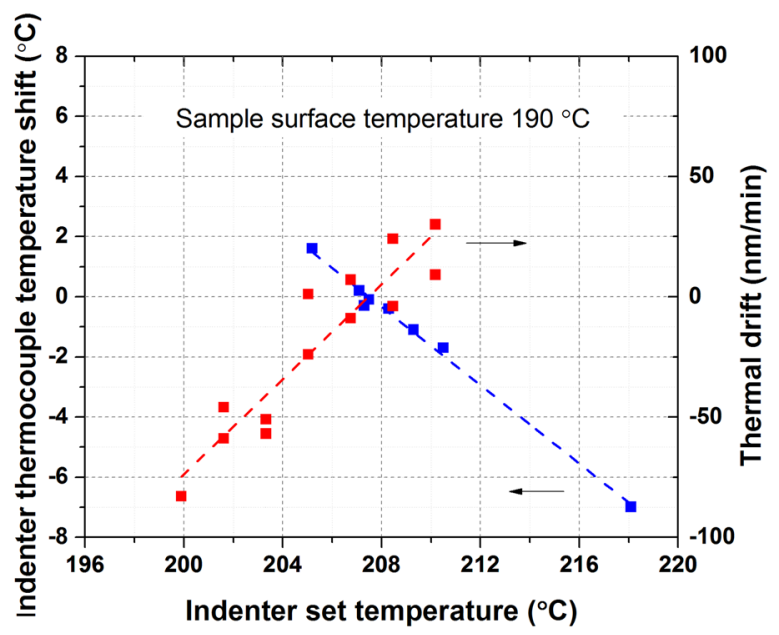

FIG. 8. (a) Temperature change of indenter thermocouple during indents at three indenter set temperatures. (b) Matching the indenter tip temperature for isothermal contact with the sample surface and achieving lowest thermal drift rates. 
active surface referencing, the reference tip approaches the sample surface first and establishes a pre-set low contact load before the indenter approaches. All these three are considered as part of the indenter approach segment resulting in a longer duration for this segment. Due to the larger thermal mass of the sample and due to changes in the infrared bath conditions during the approach, the indenter temperature changes slightly during the approach segment as well.

For calibration purposes, the change in indenter thermocouple temperature is considered during the actual indentation, i.e., the difference in temperatures between the end of the approach segment and the beginning of indenter retraction. By cycling the indenter temperature while holding the sample temperature constant, the iso-thermal condition for contact can be determined which corresponds to zero indenter thermocouple temperature change during indentation, as shown in Fig. 8. A similar contact drift minimization procedure was also followed for the reference tip.

The success of the above mentioned procedure depends on the indenter thermal mass, thermocouple sensitivity, placement of the thermocouple close to the tip, sample and indenter thermal conductivity, and efficiency of heat transfer across the sample-indenter interface. In cases where this calibration procedure might not work, the direction and magnitude of thermal drift rates can also be measured as a function of indenter temperature [Fig. 8(b)]. The thermal drift rate was calculated by applying a linear fit to the last $75 \%$ of the displacement data (as a function of time) obtained at a $2 \mathrm{mN}$ hold segment. Indenter thermocouple temperature changes and thermal drift rates show remarkable agreement with each other in terms of calculating the indenter temperature for iso-thermal contact.

\section{Thermal gradient calibration curves for indenter and reference}

Once the contact drift minimization procedure is performed on a conductive sample (copper in this case) across different test temperatures, the data can be used to determine the high temperature calibration curves for both the indenter and the reference. The sample surface temperature can be optionally read directly by a thermocouple attached between the indenter and the reference. This thermocouple remains in contact with the sample surface during

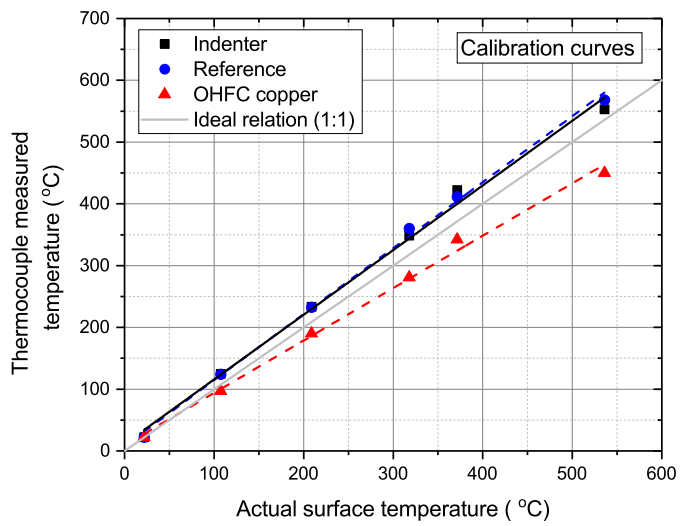

FIG. 9. High temperature calibration curves for the indenter, reference and the tested Cu sample. indentation. The iso-thermal data points across the tested temperature range and the corresponding sample surface temperatures can be used to "thermally calibrate" new indentation (and reference) tips.

Figure 9 shows the high temperature calibration curves for the indenter and the reference. Due to similar architectures and designs, the calibration curves for both the indenter and reference super-impose nicely. It is expected that new tips of similar architectures and the same tip material will yield similar results. This highlights the advantage of the high temperature tip assembly design of $\mathrm{UNHT}^{3} \mathrm{HTV}$ and reduces variability in thermal calibration data for different tips, as reported previously for tip designs based on resistive heating embedded in cement paste. ${ }^{21}$ Also, it makes it unnecessary to thermally calibrate all new tips.

\section{VALIDATION RESULTS ON DIFFERENT MATERIALS}

After having established the high temperature calibration and experimentation protocols, it is necessary to validate the developed $\mathrm{UNHT}^{3}$ HTV nanoindentation system on standard reference materials. Indents were performed using the experimental protocols outlined in Sec. IV B on copper and fused silica samples (Goodfellow Cambridge Ltd., UK). In this section, the extracted mechanical properties, hardness, and indentation modulus, obtained at elevated test temperatures, are compared with the literature. In addition, thermal drift rates at elevated temperatures are also reported.

\section{A. Copper}

Oxygen free high conductivity copper (OFHC Cu) was metallographically polished and annealed to $600{ }^{\circ} \mathrm{C}$ for $5 \mathrm{~h}$ in the nanoindentation vacuum chamber. Copper was selected due to its very high thermal conductivity $\left(385 \mathrm{Wm}^{-1} \mathrm{~K}^{-1}\right.$ ) among metals. This represents the worst case scenario for materials testing since a small thermal mismatch will result in large drift rates. Also, $\mathrm{Cu}$ has nearly vertical unloading curves which affect the extracted moduli values in the case of thermal drift. Hence, the tolerance for extracting accurate indentation moduli values for $\mathrm{Cu}$ using a diamond tip at elevated temperatures is very small.

Figure 10(a) shows representative load-displacement curves obtained on copper for various test temperatures. Due to the low melting point of copper, the upper temperature limit for testing was fixed at approximately $500{ }^{\circ} \mathrm{C}$. Unlike fast and shallow indents reported by Trenkle et al. ${ }^{27}$ to limit the influence of drift on nanomechanical property measurements, the data reported here correspond to standard quasi-static tests typical for nanoindentation. The indentation load profile was identical to that reported in Sec. IV B that spanned over 2 min of contact. A minimum of 15 indents were performed at each temperature. Due to temperature matching of the indenter, the reference, and the sample surface, the minimum achievable thermal drift rate remains low and $<2 \mathrm{~nm} / \mathrm{min}$ over the entire tested temperature range [Fig. 10(b)]. Corresponding indentation contact areas varied from $\sim 150 \mu \mathrm{m}^{2}$ at RT to $\sim 500 \mu \mathrm{m}^{2}$ at $500{ }^{\circ} \mathrm{C}$. This is a fairly large contact area and contact time on a highly thermally conductive sample. The lower envelope of thermal drift data, which mostly remains within $2 \mathrm{~nm} / \mathrm{min}$, confirms the stability of the instrument for high temperature tests.

Load-displacement curves with low thermal drift rates enable accurate extraction of indentation modulus and hardness values 


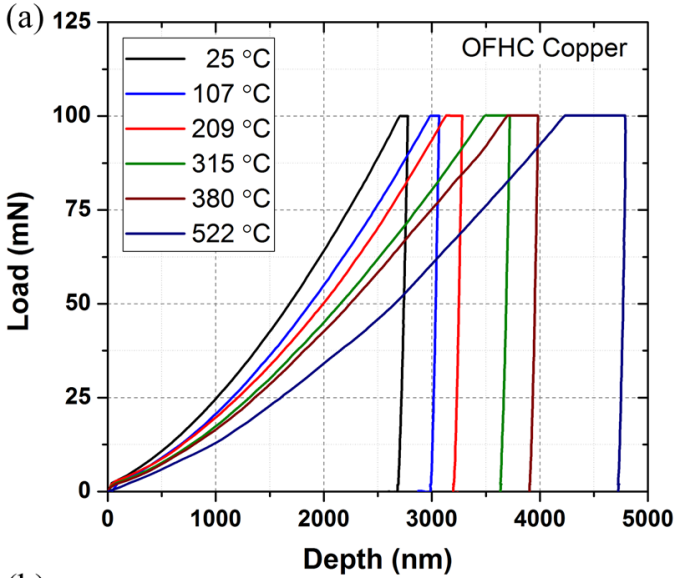

(b)

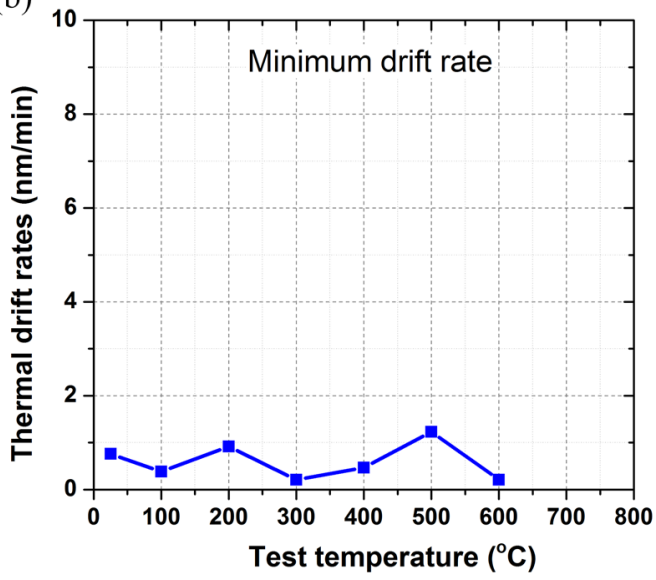

FIG. 10. (a) Compliance corrected representative load-displacement curves for copper from RT to $500^{\circ} \mathrm{C}$. Actual sample surface temperatures are reported. (b) The minimum achieved thermal drift rates from high temperature nanoindentation testing on copper using the experimental protocol outlined in this manuscript is presented.

using the method outlined by Oliver and Pharr, ${ }^{1,2}$ shown in Fig. 11. Corresponding literature data ${ }^{27,49-51}$ are also shown to aid comparison. The variation in indentation modulus and hardness follows similar trends with temperature as reported in the literature. The offset in copper modulus values arises due to anisotropy in elastic constants of copper. This results in large differences in Young's modulus of copper based on orientation with literature reported scatter being 59-77 GPa in the (001) direction, 160-202 GPa in the (111) direction, and $121-138 \mathrm{GPa}$ in the (110) direction. ${ }^{52,53}$ The hardness data lie within the various hardness ranges reported in the literature. Since hardness depends on grain size, literature data show a large variation in measured hardness values. The standard deviation in the reported mechanical properties remains similar over the entire tested temperature range, suggesting no change in instrument performance and reliability of mechanical property measurements at high temperatures. This provides the ultimate validation of the $\mathrm{UNHT}^{3} \mathrm{HTV}$ instrument for high temperature vacuum nanoindentation measurements.
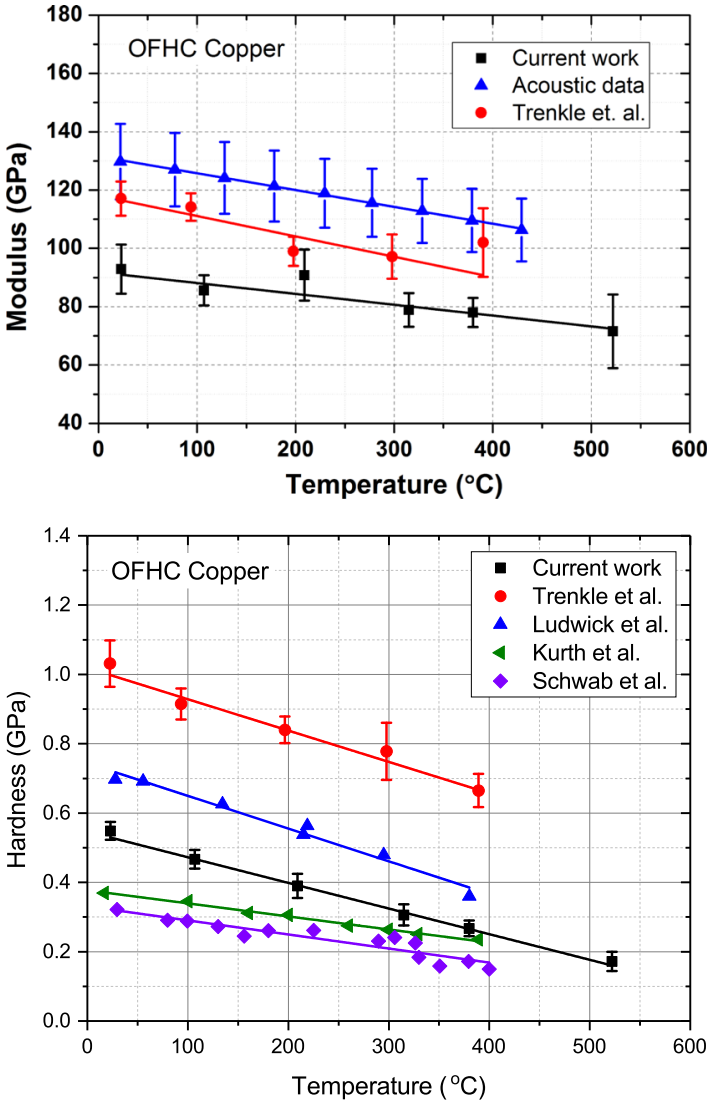

FIG. 11. Modulus and hardness of copper as a function of temperature. Literature data ${ }^{27,49-51}$ for copper are shown for comparison. Modulus data from the present work and that of Trenkle et al. are nanoindentation modulus values, while the acoustic data represent Young's modulus values.

\section{B. Fused silica}

Fused silica is a standard reference material used for room temperature calibrations and measurement verification. Due to its deformation by densification, indentation modulus and hardness do not exhibit depth dependent size effects. This makes it an ideal calibration material for nanoindentation for tip area determination and compliance calibration at room temperature. In contrast to copper, fused silica is a poor thermal conductor $\left(1.4 \mathrm{Wm}^{-1} \mathrm{~K}^{-1}\right)$. Hence, it lies at the other end of the thermal conductivity spectrum when it comes to high temperature calibration and tip-sample surface temperature tuning where temperature changes detectable by the indenter thermocouple will be very small. On the other hand, the thermal drift rates will be much lower and the window for performing low thermal drift indents will be larger.

A $5 \mathrm{~mm}$ thick fused silica sample was used for high temperature nanoindentation measurements in this study. Due to low thermal conductivity, there were large temperature gradients across the thickness of the sample. In spite of heating the sample holder to $600{ }^{\circ} \mathrm{C}$, the maximum attainable surface temperature was only $330{ }^{\circ} \mathrm{C}$. This is the highest test temperature reported for fused silica here. 



FIG. 12. Modulus and hardness of fused silica as a function of temperature determined from high temperature nanoindentation measurements. Literature data $20,21,27,54,55$ are shown for comparison. Except for NIST database that reports Young's modulus, all other modulus datasets are nanoindentation modulus values.

Figure 12 shows the extracted indentation moduli and hardness values as a function of temperature along with literature data $^{20,21,27,54,55}$ also determined by nanoindentation. The scatter in experimental data results from different grades of fused silica used. Another source of difference is that except for the measurements reported by Wheeler and Michler, ${ }^{21}$ all other studies have not explicitly reported the actual surface temperature of the tested specimen. Instead, the sample holder temperature or the sample bottom surface temperatures have been reported. Due to low thermal conductivity, possibly the temperature axis is not correct for these studies. In spite of these differences, the determined modulus and hardness data are in good agreement with literature values. This again reiterates the accuracy of high temperature measurements using the reported nanoindentation system.

\section{Alumina $\left(\mathrm{Al}_{2} \mathrm{O}_{3}\right)$ coating}

An industrially obtained alumina coating $\left(\mathrm{Al}_{2} \mathrm{O}_{3}\right)$ deposited on a tungsten carbide (WC) substrate was tested at different temperatures. The thickness of the coating was about $8-10 \mu \mathrm{m}$, and the maximum indentation depth of $\sim 400 \mathrm{~nm}$ was chosen to avoid
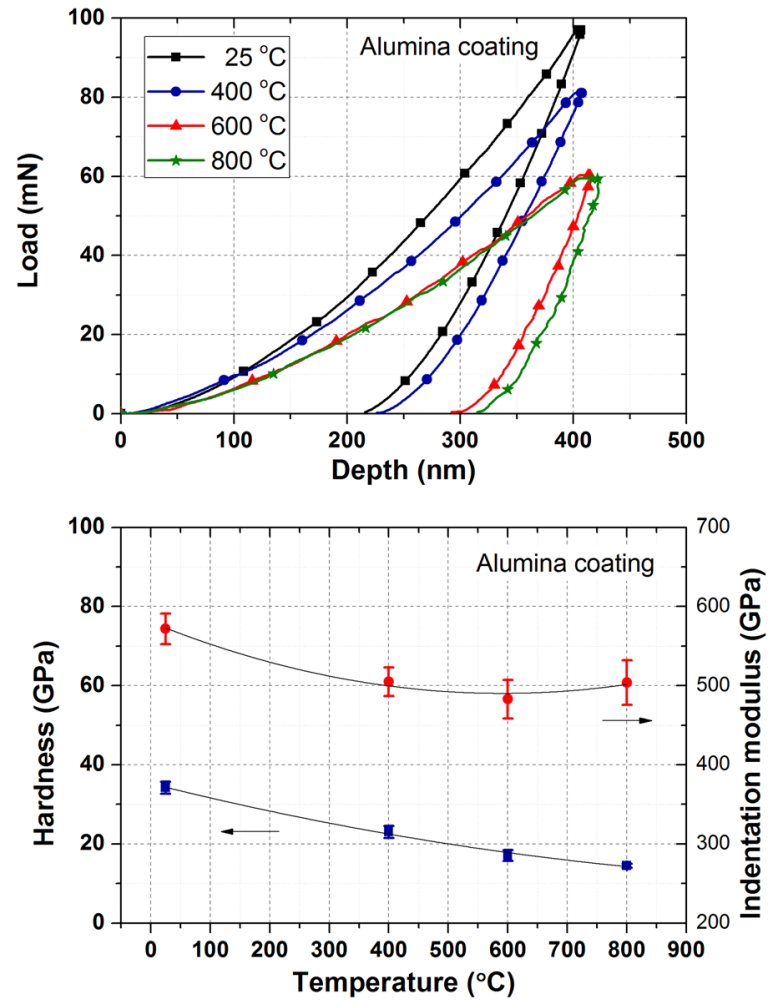

FIG. 13. Elastic modulus $E$ and hardness $H$ of alumina coating at different temperatures.

any substrate effects. ${ }^{56}$ The indentations were performed in load controlled mode with a maximum set displacement of $400 \mathrm{~nm}$ before the onset of hold segment. Since the specimen starts creeping at elevated temperatures, the maximum indentation depth fluctuates between 405 and $415 \mathrm{~nm}$. The sample was tested at four temperatures-RT, 400, 600, and $800{ }^{\circ} \mathrm{C}$. The load-displacement curves as well as the determined hardness and modulus values are shown in Fig. 13. The load-displacement curves remain smooth over the entire temperature range except at the highest temperatures. This is possibly due to the porosities present in the material. The indentation modulus drops by about $12 \%$ from room temperature to $400{ }^{\circ} \mathrm{C}$ and thereafter remains constant up to $800{ }^{\circ} \mathrm{C}$. Hardness, on the other hand, drops over the entire tested temperature range.

\section{CONCLUSIONS}

A novel high temperature vacuum nanoindentation system (UNHT ${ }^{3} \mathrm{HTV}$ ) capable of performing measurements up to $800{ }^{\circ} \mathrm{C}$ has been developed. It is based on active surface referencing and non-contact heating of the tips. Infrared heating is a proven technique and allows fine temperature tuning, thanks to the almost zero thermal inertia of the heaters. Detailed instrumental design and considerations for reliable high temperature measurements have been reported in this work. The system was adequately characterized for noise and thermal stabilization. Due to fast thermal stabilization 
times, the instrument enables high throughput testing to be performed at multiple temperatures within hours.

Extensive thermal contact drift minimization protocols have been developed and reported in this work. Temperature tuning of the tip apex and the sample surface matches well with the thermal drift minimization procedure. Both or either of these two methods can be used to minimize thermal drift while testing different materials. By following the outlined thermal drift minimization procedure, it is possible to reduce drift rates down to $2 \mathrm{~nm} / \mathrm{min}$ for Cu even at high testing temperatures. High temperature nanoindentation data have been reported on standard materials such as $\mathrm{Cu}$ and fused silica to validate the instrument. The extracted modulus and hardness values are in good agreement with literature data. This highlights the reliability and accuracy of the high temperature nanoindentation system reported here.

Due to robust design and system enhancements, it is possible to further push the testing temperatures higher than $800{ }^{\circ} \mathrm{C}$. More importantly, for the first time, this system provides the ability to perform long term, stable, and low drift measurements. This paves the way for indentation creep and other long duration tests in nanoindentation.

\section{ACKNOWLEDGMENTS}

The authors would like to acknowledge NanoHot grant from Commission for Technology and Innovation (CTI), Switzerland. The authors would also like to thank D. Frey from Empa for help with data analysis, S. Hostettler from Synton-MDP AG, Nidau, Switzerland, for joint development of high temperature indenter tips, and Christophe Tromas from Pprime Institute, University of Poitiers, France, for contributing Fig. 1 of this work.

\section{REFERENCES}

${ }^{1}$ W. C. Oliver and G. M. Pharr, J. Mater. Res. 7, 1564 (1992).

${ }^{2}$ W. C. Oliver and G. M. Pharr, J. Mater. Res. 19, 3 (2004).

${ }^{3}$ J. B. Pethica, R. Hutchings, and W. C. Oliver, Philos. Mag. A 48, 593 (1983).

${ }^{4}$ E. T. Lilleodden, W. Bonin, J. Nelson, J. T. Wyrobek, and W. W. Gerberich, J. Mater. Res. 10, 2162 (1995).

${ }^{5}$ W. A. Bonin, U.S. patent US5553486A (1 October 1993).

${ }^{6}$ J. S. Field, U.S. patent US5067346A (10 July 1986).

${ }^{7}$ M. D. Uchic and D. M. Dimiduk, Mater. Sci. Eng. A 400-401, 268 (2005).

${ }^{8}$ M. Sebastiani, K. E. Johanns, E. G. Herbert, F. Carassiti, and G. M. Pharr, Philos. Mag. 95, 1928 (2014).

${ }^{9}$ G. Mohanty, J. M. Wheeler, R. Raghavan, J. Wehrs, M. Hasegawa, S. Mischler, L. Philippe, and J. Michler, Philos. Mag. 95, 1878 (2014).

${ }^{10}$ V. Maier, K. Durst, J. Mueller, B. Backes, H. W. Höppel, and M. Göken, J. Mater. Res. 26, 1421 (2011).

${ }^{11}$ G. Mohanty, J. Wehrs, B. L. Boyce, A. Taylor, M. Hasegawa, L. Philippe, and J. Michler, J. Mater. Res. 31, 1085 (2016).

${ }^{12}$ V. Maier, B. Merle, M. Göken, and K. Durst, J. Mater. Res. 28, 1177 (2013).

${ }^{13}$ R. Rabe, J.-M. Breguet, P. Schwaller, S. Stauss, F.-J. Haug, J. Patscheider, and J. Michler, Thin Solid Films 469-470, 206 (2004).

${ }^{14}$ A. M. Minor, E. T. Lilleodden, E. A. Stach, and J. W. Morris, MRS Proc. 695, L4.4.1 (2001).

${ }^{15}$ M. Legros, D. S. Gianola, and K. J. Hemker, Acta Mater. 56, 3380 (2008).

${ }^{16}$ P. J. Imrich, C. Kirchlechner, D. Kiener, and G. Dehm, JOM 67, 1704 (2015).
${ }^{17}$ C. Niederberger, W. M. Mook, X. Maeder, and J. Michler, Mater. Sci. Eng. A 527, 4306 (2010).

${ }^{18}$ K. Wasmer, T. Wermelinger, A. Bidiville, R. Spolenak, and J. Michler, J. Mater. Res. 23, 3040 (2008).

${ }^{19}$ A. Bhowmik, I. P. Dolbnya, T. Ben Britton, N. G. Jones, G. Sernicola, C. Walter, P. Gille, D. Dye, W. J. Clegg, and F. Giuliani, Appl. Phys. Lett. 108, 111902 (2016).

${ }^{20}$ B. D. Beake and J. F. Smith, Philos. Mag. A 82, 2179 (2002).

${ }^{21}$ J. M. Wheeler and J. Michler, Rev. Sci. Instrum. 84, 045103 (2013).

${ }^{22}$ J. M. Wheeler, D. E. J. Armstrong, W. Heinz, and R. Schwaiger, Curr. Opin. Solid State Mater. Sci. 19, 354 (2015).

${ }^{23}$ J. Chen, G. A. Bell, H. Dong, J. F. Smith, and B. D. Beake, J. Phys. D: Appl. Phys. 43, 425404 (2010).

${ }^{24}$ S.-W. Lee, L. Meza, and J. R. Greer, Appl. Phys. Lett. 103, 101906 (2013).

${ }^{25}$ L. Farran, A. R. Ennos, and S. J. Eichhorn, J. Mater. Res. 24, 980 (2009).

${ }^{26}$ M. J. Mirzaali, J. J. Schwiedrzik, S. Thaiwichai, J. P. Best, J. Michler, P. K. Zysset, and U. Wolfram, Bone 93, 196 (2016).

${ }^{27}$ J. C. Trenkle, C. E. Packard, and C. A. Schuh, Rev. Sci. Instrum. 81, 073901 (2010).

${ }^{28}$ S. Korte, R. J. Stearn, J. M. Wheeler, and W. J. Clegg, J. Mater. Res. 27, 167 (2012).

${ }^{29}$ N. M. Everitt, M. I. Davies, and J. F. Smith, Philos. Mag. 91, 1221 (2011).

${ }^{30}$ J. M. Wheeler, P. Brodard, and J. Michler, Philos. Mag. 92, 3128 (2012).

${ }^{31}$ J. M. Wheeler, R. A. Oliver, and T. W. Clyne, Diamond Relat. Mater. 19, 1348 (2010).

${ }^{32}$ J. M. Wheeler and J. Michler, Rev. Sci. Instrum. 84, 101301 (2013).

${ }^{33}$ W. Kang, M. Merrill, and J. M. Wheeler, Nanoscale 9, 2666 (2017).

${ }^{34}$ M. A. Monclús and J. M. Molina-Aldareguia, in Handbook of Mechanics of Materials (Springer Singapore, Singapore, 2018), pp. 1-29.

${ }^{35}$ Z. C. Duan and A. M. Hodge, JOM 61, 32 (2009).

${ }^{36}$ B. D. Beake, A. J. Harris, J. Moghal, and D. E. J. Armstrong, Mater. Des. 156, 278 (2018).

${ }^{37}$ J. S. K.-L. Gibson, S. Schröders, C. Zehnder, and S. Korte-Kerzel, Extreme Mech. Lett. 17, 43 (2017).

${ }^{38}$ J. Cho, Q. Li, H. Wang, Z. Fan, J. Li, S. Xue, K. S. N. Vikrant, H. Wang, T. B. Holland, A. K. Mukherjee, R. E. García, and X. Zhang, Nat. Commun. 9, 2063 (2018).

${ }^{39}$ P. S. Phani and W. C. Oliver, Acta Mater. 111, 31 (2016).

${ }^{40}$ T. E. J. Edwards, F. Di Gioacchino, G. Mohanty, J. Wehrs, J. Michler, and W. J. Clegg, Acta Mater. 148, 202 (2018).

${ }^{41}$ K. Durst and V. Maier, Curr. Opin. Solid State Mater. Sci. 19, 340 (2015).

${ }^{42}$ R. C. Jacques Woirgard and B. Bellaton, U. S. patent US7685868B2 (30 March 2010).

${ }^{43}$ J. Nohava, N. X. Randall, and N. Conté, J. Mater. Res. 24, 873 (2009).

${ }^{44}$ A. A. Volinsky, N. R. Moody, and W. W. Gerberich, J. Mater. Res. 19, 2650 (2004).

${ }^{45}$ R. A. Nevshupa, M. Conte, A. Igartua, E. Roman, and J. L. de Segovia, Tribol. Int. 86, 28 (2015).

${ }^{46}$ B. Bellaton and M. Conte, WO2016071295A1 (12 May 2016).

${ }^{47}$ B. Bellaton and M. Conte, WO2016071296A1 (12 May 2016).

${ }^{48}$ B. Bellaton and M. Conte, WO2017125466A1 (27 July 2017).

${ }^{49}$ P. Ludwik, Z. Phys. Chem. 91U, 232 (1916).

${ }^{50}$ A. Kurth, Zeits. Ver. Deut. Ing. 53, 209 (1909).

${ }^{51}$ G.-M. Schwab, "Some new aspects of the strength of alloys," Trans. Faraday Soc. 45, 385 (1949).

${ }^{52}$ D. E. J. Armstrong, A. J. Wilkinson, and S. G. Roberts, J. Mater. Res. 24, 3268 (2009).

${ }^{53}$ D. F. Nelson (Springer-Verlag, Berlin/Heidelberg, 1992).

${ }^{54}$ R. G. Munro, NIST Interagency/Internal Report No. 6853, 2002.

${ }^{55}$ C. A. Schuh, C. E. Packard, and A. C. Lund, J. Mater. Res. 21, 725 (2006).

${ }^{56}$ R. Saha and W. D. Nix, Acta Mater. 50, 23 (2002). 